\title{
Semi-Individualized Acupuncture for Insomnia Disorder and Oxidative Stress: A Randomized, Double-Blind, Sham-Controlled Trial
}

\author{
Wing-Fai Yeung $\mathbb{D}^{\prime}$ \\ Branda Yee-Man Yu ${ }^{2}$ \\ John Wai-Man Yuen' \\ Janice Yuen Shan Ho' \\ Ka-Fai Chung ${ }^{3}$ \\ Zhang-Jin Zhang ${ }^{4}$ \\ Deejay Suen Yui Mak ${ }^{5}$ \\ Lorna Kwai-Ping Suen ${ }^{6}$ \\ Lai-Ming $\mathrm{Ho}^{7}$
}

'School of Nursing, The Hong Kong Polytechnic University, The Hong Kong SAR, People's Republic of China;

2Department of Psychology, The University of Hong Kong, The Hong Kong SAR, People's Republic of China; ${ }^{3}$ Department of Psychiatry, The University of Hong Kong, The Hong Kong SAR, People's Republic of China; ${ }^{4}$ School of Chinese Medicine, The University of Hong Kong, The Hong Kong SAR, People's Republic of China; ${ }^{5}$ School of Medical and Health Sciences, Tung Wah College, The Hong Kong SAR, People's Republic of China; ${ }^{6}$ School of Nursing, Tung Wah College, The Hong Kong SAR, People's Republic of China; ${ }^{7}$ School of Public Health, The University of Hong Kong, The Hong Kong SAR, People's Republic of China

Correspondence: Wing-Fai Yeung School of Nursing, The Hong Kong Polytechnic University, Room FG429, Hung Hom, Hong Kong, People's Republic of China

Email jerry-wf.yeung@polyu.edu.hk
Background: Acupuncture is an alternative treatment for improving sleep, and it may attenuate oxidative stress, which is a possible pathophysiological factor in insomnia. The aim of this study was to examine the efficacy and safety of a semi-individualized acupuncture in improving sleep and explore its effect on oxidative stress parameters in adults with insomnia disorder.

Methods: In this randomized sham-controlled trial, 140 participants were randomly assigned to either a 4-week semi-individualized traditional acupuncture (TA) or noninvasive sham acupuncture (SA). The primary outcome measure was the sleep-diary-derived sleep efficiency. Other outcomes included sleep diary and actigraphy, Insomnia Severity Index, anxiety and depressive symptoms, and quality of life. Blood samples were taken to measure oxidative stress parameters (malondialdehyde, glutathione peroxidase, paraoxonase, and arylesterase).

Results: Although no significant difference was found in the primary outcome measure, both sleep-diary-derived and actigraphy-derived total sleep time (TST) were significantly increased in the TA group at 1-week posttreatment (mean difference in sleep diary $=22.0$ min, $p=0.01$, actigraphy $=18.8 \mathrm{~min}, p=0.02$ ). At 5-week posttreatment follow-up, a significantly higher proportion of participants in the TA group showed sleep-diaryderived sleep efficiency (SE) $\geq 85 \%$ than in the SA group $(55.6 \%$ versus $36.4 \%, p=0.03$ ). Conclusion: TA and SA did not significantly differ in improving subjective sleep efficiency in individuals with insomnia disorder. However, the TA group showed a short-term effect on improving TST as measured by both sleep diary and actigraphy at 1-week posttreatment, but there were no differences in the oxidative stress parameters.

Clinical Trial Registration: Registry: ClinicalTrials.gov; Title: Acupuncture in the Modulation of Peripheral Oxidative Stress Insomnia; Identifier NCT03447587; URL: https://clinicaltrials.gov/ct2/show/NCT03447587.

Keywords: sleep, electroacupuncture, oxidative stress, placebo, Chinese medicine, acupoints

\section{Introduction}

Insomnia is dissatisfaction with sleep quantity or quality, with difficulty initiating asleep, maintaining sleep, or early morning awakening, that occur despite adequate opportunities for sleep, and result in daytime impairment. ${ }^{1}$ Insomnia is the most prevalent sleep disorder affecting $9-15 \%$ of the adult populations. ${ }^{2}$ The prevalence of insomnia disorder was around $10.8 \%$ in Hong Kong, according to the Diagnostic and Statistical Manual of Mental Disorders, Fifth Edition (DSM-5). ${ }^{3}$ Insomnia can lead to fatigue, irritability, and impaired daytime functioning and correlates with disturbed mood. ${ }^{4,5}$ Insomnia has also been found to be associated with psychiatric disorders, substance abuse, suicide, pain, 
and cardiovascular diseases. ${ }^{6-9}$ Early diagnosis and treatment of insomnia are crucial, because if left untreated, it may contribute to the development of anxiety and depressive disorders. ${ }^{10}$

Pharmacological and psychological treatments for insomnia have proven to be effective, but these therapies imply limitations. Pharmacotherapies such as benzodiazepines and non-benzodiazepine hypnotics are associated with abuse, dependence, and they have uncertain efficacy with long-term use. ${ }^{11}$ Psychological and behavioural therapies such as cognitive behavioral therapies are effective in the long-term, ${ }^{12,13}$ but they have remained largely underused in primary care, probably due to the extensive behavioural changes required from the patients and the need for significant advanced training for effective implementation. ${ }^{14}$ Given the limitations of these conventional treatment, people with insomnia tend to seek complementary and alternative therapies. ${ }^{15}$ According to a survey, acupuncture ranked the second most commonly used complementary and alternative therapy for insomnia in Hong Kong. ${ }^{16}$

Previous systematic reviews suggested that acupuncture could be effective for insomnia, but the results have been inconclusive due to methodological shortcomings and the lack of standard measurements of sleep. ${ }^{17-21}$ A major limitation is that these studies lacked standard measurements of sleep, such as objective sleep assessment and sleep diaries, which are essential in insomnia efficacy trials. In addition, it is believed that the individualized approach is of optimal effectiveness in terms of traditional Chinese medicine (TCM) theory ${ }^{22}$ and resembles the clinical practice. However, the effect of acupuncture using an individualized protocol with sham-acupuncture treatment are inconclusive. ${ }^{17}$ Thus, more high-quality randomized controlled trials (RCTs) with rigorous design are needed to confirm the beneficial effects of acupuncture on insomnia. The results will more truly reflect the effects of acupuncture in real-world practice. The findings will enrich our knowledge about personalized medicine in TCM.

Oxidative stress, a state of imbalance between the generation of reactive oxygen species and antioxidant defenses. ${ }^{23}$ Oxidative stress has been postulated as an important factor in the pathophysiology of insomnia in that cerebral free radicals accumulate during wakefulness and are removed during sleep. ${ }^{24}$ A meta-analysis of animal studies suggested that experimental sleep deprivation promotes oxidative stress such as increasing in hippocampal lipid peroxidation and decreasing in glutathione concentration. ${ }^{25}$ Clinical studies also demonstrated that subjects with insomnia had significantly higher oxidative stress parameters compared with the good sleeper control group. ${ }^{26,27}$ A study in postmenopausal women complaining of insomnia found elevated levels of blood thiobarbituric acid reactive substances. ${ }^{28}$ These studies suggested that oxidative stress may be an underlying condition associated with insomnia and may serve as a biomarker related to insomnia and sleep loss. ${ }^{24}$ However, whether oxidative stress parameters change after interventions has not been examined.

Emerging evidence from animal studies has proposed that acupuncture is linked with redox modulation in various disorders such as vascular dementia, Alzheimer's disease, Parkinson's disease, and hypertension. ${ }^{29}$ However, clinical studies that examine the effects of acupuncture in the modulation of peripheral oxidative stress on insomnia are lacking. The present RCT aimed to examine (a) the efficacy and safety of a 4-week semiindividualized acupuncture in improving sleep and (b) explore its effects on oxidative stress parameters in adults with insomnia disorder.

\section{Methods}

\section{Trial Design}

This was a randomized, sham-control, subject- and assessor-blind trial examining the efficacy and safety of a 4-week semi-individualized acupuncture intervention on improving sleep and oxidative stress in individuals with insomnia disorder. Participants were randomized to traditional acupuncture group (TA) or sham acupuncture group (SA). Ethic approval was obtained from the review board of the Hong Kong Polytechnic University. All the participants were informed the purpose of this study and randomization procedure with a $50 \%$ of chance receiving TA or SA. The study was conducted in accordance with the Declaration of Helsinki. This trial has been registered at ClinicalTrials.gov (\#NCT03447587).

\section{Participants}

From April 2018 to September 2019, 140 participants with insomnia disorder were recruited from the community through advertisements in universities, university clinics, mass mail, and social media in Hong Kong. Eligible participants were (a) residents of Hong Kong, (b) Chinese ethnicity aged 18 to 65 , (c) having insomnia disorder 
according to the DSM-5 diagnostic criteria by means of a validated structured interview, Brief Insomnia Questionnaire, ${ }^{16}$ and (d) scoring at least 11 points on the Insomnia Severity Index (ISI), which is an optimal cutoff with $97.2 \%$ sensitivity and $100 \%$ specificity for detection of insomnia in clinical samples..$^{30,31}$

Individuals were excluded if they (a) had any current major depressive disorder, generalized anxiety disorders or panic disorder, manic or hypomanic episode, substance use disorders, organic mental disorder, schizophrenia or any other psychotic disorder as defined by DSM-IV criteria using the Structured Clinical Interview for DSM-IV (SCID); (b) had current physical illnesses that contributed significantly to their insomnia; (c) were pregnant; (d) had previously been diagnosed as having sleep apnea or periodic limb movement disorder; (e) were assessed by a Home Single-Channel Nasal Pressure (Apnealink; Resmed: Sydney, Australia) screening, which is an validation alternative to polysomnography for diagnosis of sleep apnea with an apnea-hypopnea index $>5 ;^{32}$ (f) were at significant suicidal risk according to SCID; (g) had an infection or abscess close to the site of the selected acupoints; (h) had valvular heart defects, bleeding disorders, or were taking anticoagulant drugs, used any implanted electrical device such as pacemakers, defibrillators, brain and nerve stimulators, etc.; (i) had received acupuncture treatment within 6 months; (j) were taking herbal remedies, over-the-counter medication, or psychotropic drugs for insomnia symptoms 2 weeks prior to the baseline; or (k) were shift workers. Written consents for study procedures were obtained from all participants.

\section{Intervention}

Participants in both groups received two sessions of 30 minutes' acupuncture treatment weekly for 4 consecutive weeks in a supine position in a treatment room of the School of Nursing at the Hong Kong Polytechnic University.

\section{Traditional Acupuncture (TA)}

A semi-standardized treatment protocol was adopted in the selection of acupoints, consisting of both fixed and additional acupoints for a total of 16 acupoints. Eight fixed acupoints, were selected according to the existing literature ${ }^{33-35}$ and expert consensus from our research team, including bilateral Ear-Shenmen, Touwei (ST8), Sishencong (EX-HN1), and unilateral Yintang (EXHN3) and Baihui (GV20) on the head. The selection of eight additional acupoints was based on the clinical judgement of acupuncturists, including unilateral Shenting (GV24), bilateral Taiyang (EX-HN5), Shuaigu (GB8), Toulinqi (GB15), Neiguan (PC6), Shenmen (HT7), Sanyinjiao (SP6), Taichong (LV3), and Anmian (EX-HN22). The list of suggested additional acupoints were empirical for treating insomnia based on our previous systematic review. ${ }^{36}$ Resembling the usual practice of acupuncture, the acupuncturist ascertained participants' current symptoms using a TCM symptom checklist $^{37}$ to record their manifestation, pulse, and tongue features as well as their responses to the previous treatment at the beginning of each session. Greater flexibility is therefore allowed that the selection of acupoints can be adjusted based on the acupuncturist's clinical judgement and experience. Skin around the selected acupoints was sterilized using $75 \%$ alcohol. Sterilized disposable needles (Hwato, China, $0.25 \times 30 \mathrm{~mm}$ ) were inserted at each acupoint by the acupuncturist and twisted and thrust forward and backward to achieve "De qi," which was believed to indicate effective needling in TCM theory. An electric stimulator (AWQ 104L, Electronic Acupunctoscope, Hong Kong) was connected to four pairs of needles and delivered a constant and continuous wave of electric stimulation (frequency of 4 $\mathrm{Hz}$ ). The needles were retained for 30 minutes and then removed.

\section{Sham Acupuncture (SA)}

Participants in the SA group received the same consultation and needling procedure, and number of needles as the TA group. However, Streitberger sham needles were tapped at non-acupoint sites that were $1 \mathrm{~cm}$ away to the selected acupoints. Streitberger sham needles, validation non-invasive devices, ${ }^{38}$ produced similar pricking sensation to TA without causing skin penetration. Needles were also connected to an electric stimulator with zero amplitude. For the purpose of disguise, participants were told that the electric stimulator was set at a fixed level and advised to inform the acupuncturist if they felt the impulse was too strong.

\section{Treatment Fidelity}

Three registered Chinese medicine practitioners with at least five years of clinical experience delivered treatments. The first 10 acupuncture sessions were inspected by the principal investigator with a pre-designed checklist, and 
some random visits were made to assess the adherence to the treatment protocol.

\section{Outcome Measures}

All the outcomes were assessed at baseline, 1-week posttreatment (Week 5) and 5-week posttreatment (Week 9) for immediate and short-term effect. Objective measures of oxidative stress were assessed only at baseline and 1-week posttreatment. The acupuncturists were not involved in data collection. Participants were asked to guess the grouping (TA or SA) to assess the success of subject blinding at the end of the last acupuncture session. Outcome assessments and data analysis were conducted by a research assistant who was blinded to the group allocation.

\section{Subjective Measures}

Sleep diary ${ }^{39}$ was used in the present study to record the daily variations of sleep-wake patterns for 7 consecutive days, allowing the estimation of sleep-onset latency (SOL), wake after sleep onset (WASO), sleep efficiency (SE), total sleep time (TST) and total time in bed (TIB). The sleep-diary derived SE which was calculated by dividing TST by TIB, was the primary subjective outcome of efficacy. Subjective sleep diary outcome was chosen to be the primary outcome since the diagnosis of insomnia is also based on the subjective experience of sleep disturbances. ${ }^{1}$ The sleep-diary-derived sleep parameters other than SE were included as secondary outcomes. The Insomnia Severity Index (ISI) is a 7-item scale assessed the perceived severity of insomnia symptoms. ${ }^{30}$ The anxiety and depression subscales of the Hospital Anxiety and Depression Scale (HADS) evaluated the level of anxiety/ depressive symptom. ${ }^{40}$ The Short-Form Six-Dimensions (SF6D) is a preference-based index of the health-related quality of life, and its scores range from 0 to $1 .^{41}$ The norms of the SF6D scores in the Hong Kong population with mental illness were $0.674(95 \% \mathrm{CI}=0.651,0.697){ }^{42}$ The validated Chinese version of ISI, ${ }^{43} \mathrm{HADS}^{44}$ and SF6D ${ }^{45}$ were used in this study.

\section{Objective Measures}

Venous blood samples were collected by venipuncture technique from 8 a.m. to 9 a.m. following an overnight fasting period. The oxidative stress parameters include malondialdehyde (MDA), and also the activities of glutathione peroxidase (GPx), Paraoxonase (PON), and Arylesterase (ARE). MDA, a commonly used marker of lipid peroxidation and an indicator of oxidative stress, was measured using a Cayman thiobarbituric acid reactive substances assay kit (Cat. No. 10009055). GPx enzyme activity was determined with a Glutathione Peroxidase Cellular Activity Assay from Sigma Chemicals (Cat. No. CGP1). Concentrations of human PON were measured using a commercial ELISA Kit (Cusabio; Cat. No. CSB-E09484h). ARE activity was determined using a commercial arylesterase/paraoxonase assay (Zeptometrix Corporation, Buffalo, NY, USA; Cat. No. 0801199).

Objective sleep data was collected by wrist actigraphy. ${ }^{46}$ Participants were told to wear a wrist actigraphy to estimate sleep parameters by measuring physical movements (Motionlogger Micro Watch; Ambulatory Monitoring, Inc., Ardsley, NY, USA), on their non-dominant wrist for 7 consecutive nights, the same period indicated in the selfcompleted sleep diary. If the subjects with more than 2 days of missing data in actigraphy or sleep diary, then the actigraphy or sleep diary outcome would be considered as missing because not able to capture the day-to-day variation of sleep across a week.

\section{Assessment of Safety}

Adverse events were monitored and recorded at every visit using a standardized adverse event record form for acupuncture. $^{47}$ Reasons for withdrawal were recorded in detail and any serious adverse events were followed up until the resolution of symptoms.

\section{Assessment of Credibility}

The Credibility of Treatment Rating Scale (CTRS) was used to assess the treatment expectancy of the received treatment with a 6-point Likert scale. ${ }^{48}$ Scores of CTRS were reversed to indicate the level of confidence and expectation of efficacy towards the treatment from 1 (the least) to 6 (the most). The CTRS was assessed at baseline and the second and last treatment sessions.

\section{Sample Size}

The targeted sample size was calculated with a statistical power of $80 \%$ and a statistical significance level at 0.05 to detect a between-groups effect size of 0.64 in the sleepdiary-derived SE, which was estimated from previous sham-controlled RCTs of acupuncture for insomnia. ${ }^{34}$ With an estimated attrition rate of $15 \%$ at 5 -week posttreatment, a total sample of 140 was determined. 


\section{Randomization, Allocation Concealment, and Blinding}

A random block size of four to six was adopted to allocate all eligible participants randomly to either the TA or SA group, which were equally sized. A computer-generated randomization list was prepared and enclosed in opaque, sealed, and sequentially numbered envelopes. The sealed envelopes were opened by the acupuncturist only when the participants had completed all baseline assessments.

\section{Data Analysis}

SPSS 25.0 (IBM Corporation, Armonk, NY, USA) was adopted for data analyses. Actigraphy data was analyzed with Action-W software (Ambulatory Monitoring, Inc., Ardsley, NY, USA). Group comparisons of SE by sleep diary, oxidative stress parameters, and other outcome measures at each time point were performed using a linear mixed-effects model. Effect size (ES) was determined as Cohen's $d$ with the mean differences divided by the pooled standard deviations. ${ }^{49}$ Analyses were performed with both the intention-to-treat and per-protocol approach. A completer analysis was performed to include those who had received at least six acupuncture treatment sessions. The clinical significance of the interventions was estimated by the proportion of participants who reached sleep-diary-derived SOL or WASO of 30 mins or less, and SE of at least $85 \%$. Logistic-regression analyses were used to compare the proportions of each group meeting these clinical significance criteria.

\section{Results \\ Study Participants}

The participants' characteristics and baseline assessment are presented in Table 1. One hundred and forty participants were allocated evenly to the TA and SA groups (70 participants in each group, Figure 1). The 140 participants had an average age of 42.1 years $(\mathrm{SD}=13.0)$ and $80 \%$ were female. At baseline, the mean score of ISI was 16.0 $(\mathrm{SD}=3.6)$, indicating a moderate insomnia severity. No significant between-group differences were observed in their demographic factors and baseline assessments (all $p>0.05$, Table 1) except for a shorter TST measured by actigraphy in the TA group $(p=0.04)$.

\section{Primary Outcome}

The differences in the sleep-diary-derived SE of the TA and SA groups across study time-points were compared using a mixed-effects model (Table 2). The TA and SA groups did not significantly differ in SE by sleep diary at 1 week or 5 weeks posttreatment (all $p>0.05$ ), although both groups showed a trend of increase in sleep diary-derived SE from baseline to 1-week and 5-week post-treatment.

\section{Secondary Outcome}

The mixed-effects model showed that the TA group had a longer TST as measured by both sleep diary $(d=0.43$, $p=0.01)$ and actigraphy $(d=0.40, p=0.02)$ when compared to the SA group at 1 week posttreatment. In view of the significant baseline imbalance in the actigraphy-derived TST, a mixed-effects model analysis with an adjusted baseline difference was performed and the results remained significant $(p=0.04)$. There was no significant between-group difference in other subjective (Table 2) and objective sleep parameters (Table 3 ), or in other secondary measures (all $p>0.05$, Table 4).

\section{Oxidative Stress Parameters}

The between-group difference in oxidative stress markers was explored (Table 5). SA groups showed a significant decrease in MDA level after the intervention compared to the TA group $(p=0.02)$. Although there was a trend of decrease in ARE and GPx activities, there was no significant between-group difference in the measured oxidative stress parameters after the intervention (all $p>0.05$ ).

\section{Clinical Significance}

The proportions of participants with clinically significant improvements were compared between the TA and SA groups (Supplementary Table 1). A significantly higher proportion of participants in the TA group showed sleepdiary-derived $\mathrm{SE} \geq 85 \%$ than the SA group at 5-week posttreatment $(55.6 \%$ vs $36.4 \%, p=0.03)$. No significant difference was observed in the proportion of participants reporting sleep-diary-derived SOL or WASO $\leq 30$ minutes (all $p>0.05$ ).

\section{Adverse Events}

Regarding safety (Supplementary Table 2), during the 4-week treatment period, the most commonly reported adverse events were pain at needling sites $(\mathrm{n}=18$, $12.9 \%)$, bruising $(\mathrm{n}=13,9.3 \%)$ around acupuncture sites, and dizziness pain $(\mathrm{n}=7,5.0 \%)$. Most of the reported adverse events were mild in severity. Only bruising and bleeding around acupuncture sites were more frequently reported in the TA during the treatment period 
Table I Socio-Demographic and Clinical Characteristics of Study Participants

\begin{tabular}{|c|c|c|c|c|}
\hline Variables $^{\mathbf{a}}$ & $\begin{array}{l}\text { All Sample } \\
(\mathrm{N}=\mid \mathbf{4 0})\end{array}$ & $\begin{array}{c}\text { TA } \\
(N=70)\end{array}$ & $\begin{array}{c}\text { SA } \\
(N=70)\end{array}$ & $P^{\mathbf{b}}$ \\
\hline Age, years & $42.1 \pm 13.0$ & $42.2 \pm 12.8$ & $42.0 \pm 13.3$ & 0.92 \\
\hline Female & $112(80.0)$ & $56(80.0)$ & $56(80.0)$ & 1.00 \\
\hline BMI, $\mathrm{kg} / \mathrm{m}^{2}$ & $21.1 \pm 2.5$ & $21.2 \pm 2.3$ & $21.0 \pm 2.6$ & 0.50 \\
\hline Married & $67(47.9)$ & $34(48.6)$ & $33(47.1)$ & 0.87 \\
\hline College attainment & $94(67.1)$ & $47(67.1)$ & $67(67.1)$ & 1.00 \\
\hline Being employed & $78(55.7)$ & $39(55.7)$ & $39(55.7)$ & 1.00 \\
\hline Higher family income & $70(50.0)$ & $30(42.9)$ & $40(57.1)$ & 0.09 \\
\hline Insomnia duration, years & $5.3 \pm 6.1$ & $5.0 \pm 5.8$ & $5.5 \pm 6.4$ & 0.62 \\
\hline History of psychiatric disorder & $16(11.4)$ & $8(11.4)$ & $8(I I .4)$ & 1.00 \\
\hline \multicolumn{5}{|l|}{ Previous treatment(s) seek for insomnia } \\
\hline Sleep medication & $33(23.6)$ & $15(21.4)$ & $18(25.7)$ & 0.55 \\
\hline Acupuncture/acupressure & $22(15.7)$ & $10(14.3)$ & $12(17.1)$ & 0.64 \\
\hline Chinese medicine & $54(38.6)$ & $31(44.3)$ & $23(32.9)$ & 0.17 \\
\hline \multicolumn{5}{|l|}{ Sleep parameters measured by sleep dairy } \\
\hline $\mathrm{SOL}, \min$ & $36.7 \pm 22.9$ & $33.2 \pm 19.1$ & $40.3 \pm 25.9$ & 0.07 \\
\hline WASO, min & $44.6 \pm 43.1$ & $44.5 \pm 36.8$ & $44.6 \pm 48.8$ & 0.99 \\
\hline TST, min & $341.8 \pm 71.2$ & $34 I .7 \pm 74.1$ & $341.8 \pm 68.7$ & 0.99 \\
\hline SE, \% & $74.3 \pm 14.4$ & $75.3 \pm 14.0$ & $73.3 \pm 14.8$ & 0.42 \\
\hline \multicolumn{5}{|l|}{ Sleep parameters measured by actigraphy } \\
\hline SoL, min & $21.8 \pm 13.5$ & $20.0 \pm 9.7$ & $23.5 \pm 16.3$ & 0.12 \\
\hline WASO, min & $34.6 \pm 29.3$ & $35.2 \pm 28.4$ & $34.1 \pm 30.4$ & 0.83 \\
\hline TST, $\min$ & $404.1 \pm 52.7$ & $394.9 \pm 55.5$ & $4 \mid 3.3 \pm 48.4$ & 0.04 \\
\hline $\mathrm{SE}, \%$ & $85.0 \pm 7.3$ & $84.7 \pm 7.2$ & $85.3 \pm 7.6$ & 0.66 \\
\hline ISI, 0-28 & $16.0 \pm 3.6$ & $16.3 \pm 3.3$ & $15.7 \pm 3.8$ & 0.31 \\
\hline Anxiety assessed by HADS, $0-28$ & $8.2 \pm 3.4$ & $8.1 \pm 3.7$ & $8.2 \pm 3.1$ & 0.77 \\
\hline Depression assessed by HADS, 0-28 & $6.4 \pm 3.5$ & $6.4 \pm 3.4$ & $6.5 \pm 3.7$ & 0.81 \\
\hline SF-6D utility score, $0-I$ & $0.69 \pm 0.12$ & $0.70 \pm 0.13$ & $0.69 \pm 0.12$ & 0.79 \\
\hline \multicolumn{5}{|l|}{ CTRS, $1-6^{c}$} \\
\hline Confidence in effectiveness & $4.3 \pm 0.8$ & $4.3 \pm 0.8$ & $4.3 \pm 0.9$ & 0.92 \\
\hline Confidence in recommending to others & $4.4 \pm 0.9$ & $4.5 \pm 0.8$ & $4.4 \pm 0.9$ & 0.70 \\
\hline Perception of treatment rationale & $4.7 \pm 0.8$ & $4.7 \pm 0.8$ & $4.7 \pm 0.9$ & 0.92 \\
\hline Likelihood of relieving other complaints & $4.2 \pm 0.9$ & $4.3 \pm 0.9$ & $4.2 \pm 0.9$ & 0.51 \\
\hline \multicolumn{5}{|l|}{ Oxidative stress markers by fasting blood } \\
\hline Paraoxonase (PON), mlU/mL & $1063.8 \pm 300.6$ & $1060.1 \pm 300.4$ & $1067.4 \pm 303.0$ & 0.89 \\
\hline Malondialdehyde (MDA), uM & $2.7 \pm 1.6$ & $2.5 \pm 1.3$ & $2.8 \pm 1.8$ & 0.18 \\
\hline Arylesterase (ARE), kU/L & $41.8 \pm 19.1$ & $42.0 \pm 19.9$ & $41.7 \pm 18.4$ & 0.92 \\
\hline Glutathione Peroxidase (GPx), Units/mL & $0.8 \pm 0.4$ & $0.9 \pm 0.4$ & $0.8 \pm 0.4$ & 0.52 \\
\hline
\end{tabular}

Notes: ${ }^{a}$ Data are presented as mean \pm standard deviation or number (\%). 'Independent $t$-test or chi-square was used for comparison. As all differences were due to randomization (ie chance), $P$ values are for reference only. ${ }^{C}$ Higher score indicated higher credibility towards treatment received.

Abbreviations: BMI, Body Mass Index; CTRS, Credibility of Treatment Rating Scale; HADS, Hospital Anxiety and Depression Scale; ISI, Insomnia Severity Index; SA, Shamacupuncture; SE, Sleep Efficiency; SF-6D, Short-Form Six-Dimension health survey; SOL, Sleep Onset Latency; TA, Traditional-acupuncture; TST, Total Sleep Time; WASO, Wake After Sleep Onset.

( $p<0.001$ and $p=0.03$, respectively). No difference in the frequency of adverse events was reported in the 5 -week posttreatment follow-up visits (all $p>0.05$ ). Withdrawal due to AE was $1.4 \%(\mathrm{n}=1)$ for both the TA and SA, which was not significantly different $(p=1.00)$. No serious adverse event was reported. 


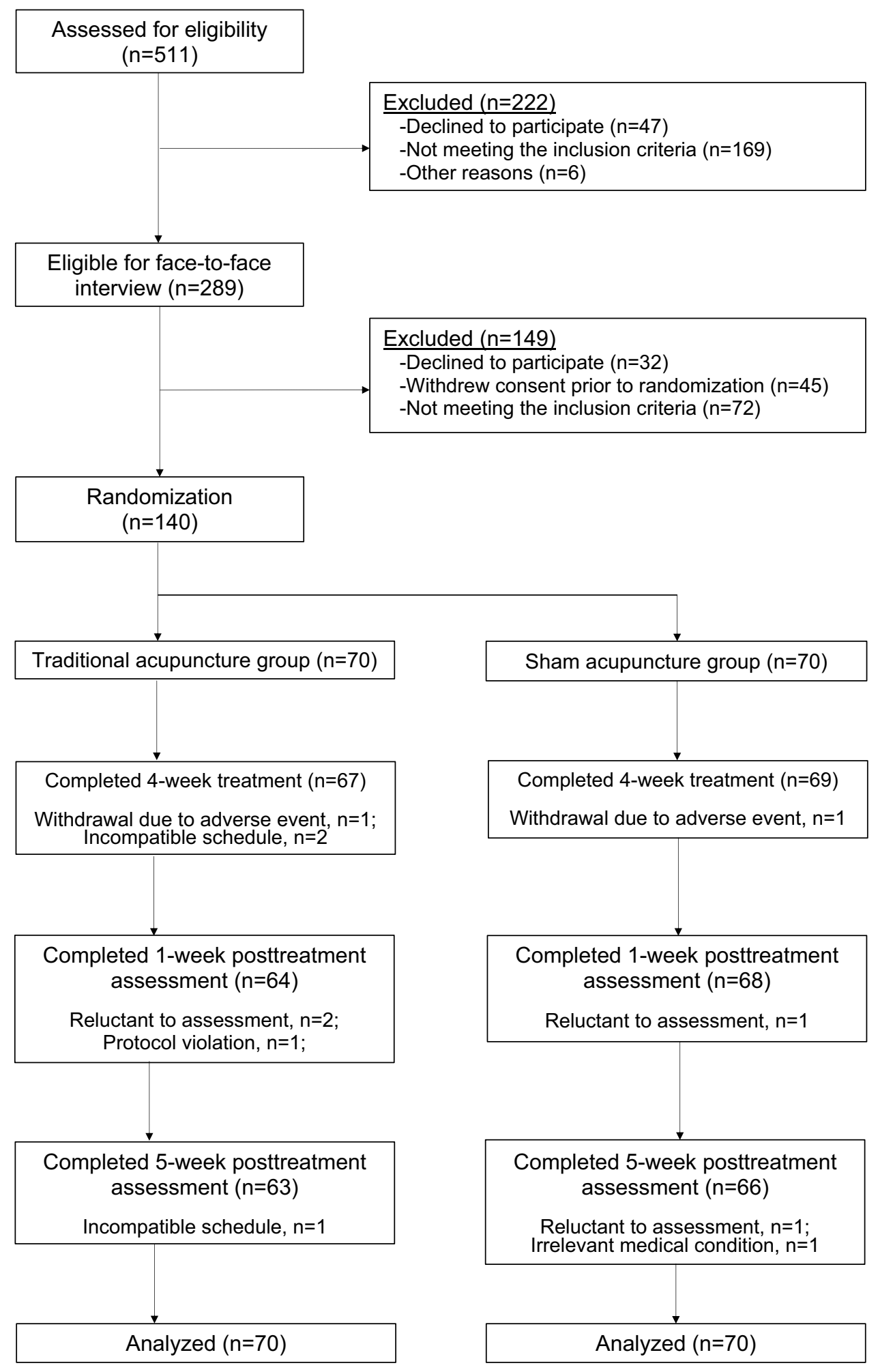

Figure I Study recruitment flowchart. 


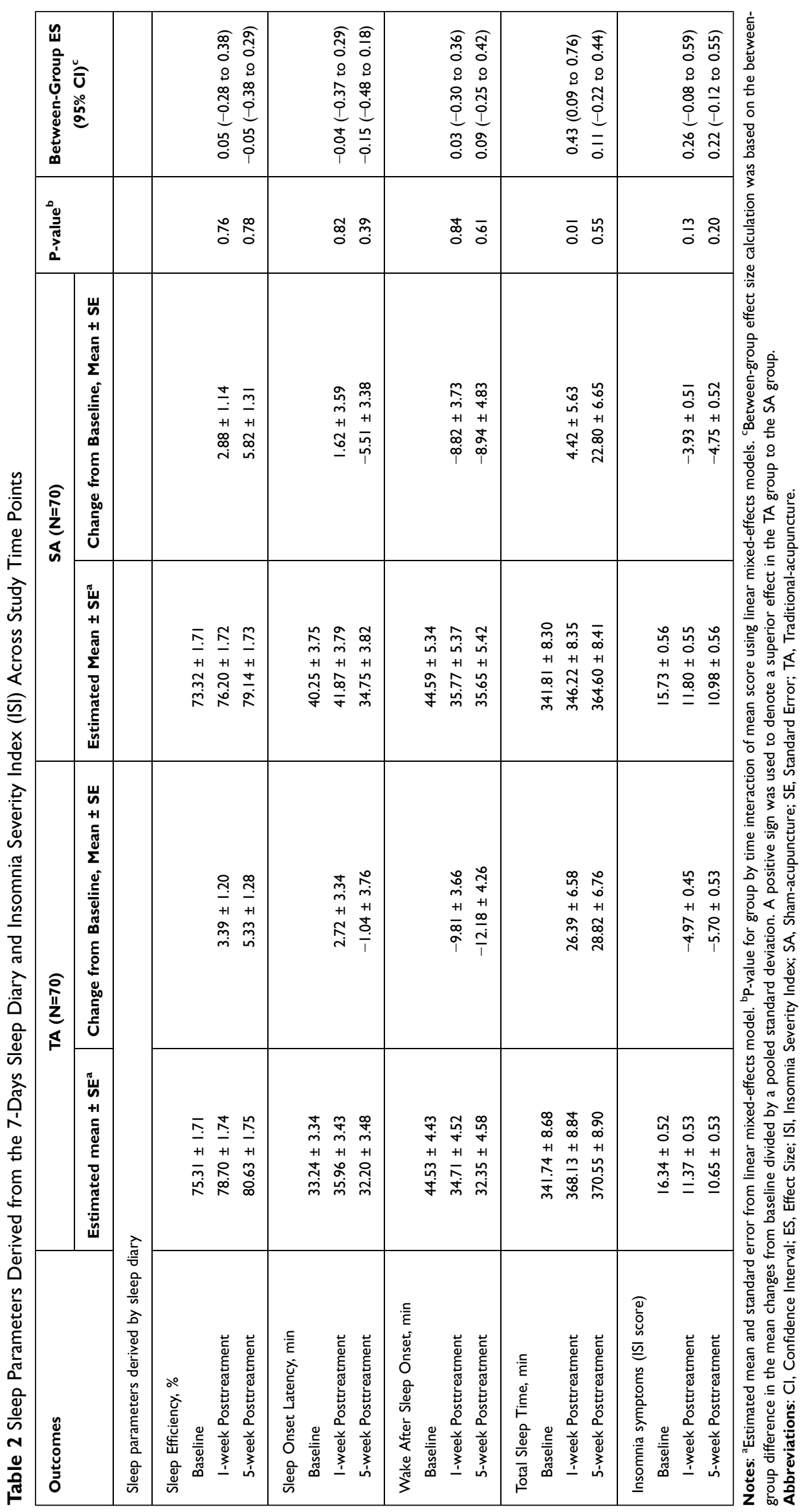


Table 3 Sleep Parameters Derived from Actigraph Across Study Time Points

\begin{tabular}{|c|c|c|c|c|c|c|}
\hline \multirow[t]{2}{*}{ Outcomes } & \multicolumn{2}{|c|}{ TA $(N=70)$} & \multicolumn{2}{|c|}{ SA $(N=70)$} & \multirow[t]{2}{*}{$P$-value ${ }^{b}$} & \multirow{2}{*}{$\begin{array}{c}\text { Between-Group ES } \\
(95 \% \mathrm{Cl})^{\mathrm{c}}\end{array}$} \\
\hline & $\begin{array}{l}\text { Estimated } \\
\text { Mean } \pm \mathrm{SE}^{\mathrm{a}}\end{array}$ & $\begin{array}{c}\text { Change from Baseline, } \\
\text { Mean } \pm \text { SE }\end{array}$ & $\begin{array}{l}\text { Estimated } \\
\text { Mean } \pm \mathbf{S E}^{\mathrm{a}}\end{array}$ & $\begin{array}{c}\text { Change from Baseline, } \\
\text { Mean } \pm \text { SE }\end{array}$ & & \\
\hline \multicolumn{7}{|c|}{ Sleep parameters derived by actigraphy } \\
\hline \multicolumn{7}{|l|}{ Sleep Efficiency, \% } \\
\hline Baseline & $84.74 \pm 0.84$ & & $85.29 \pm 0.84$ & & & \\
\hline I-week Posttreatment & $85.50 \pm 0.86$ & $0.76 \pm 0.68$ & $85.95 \pm 0.84$ & $0.66 \pm 0.57$ & 0.92 & $0.02(-0.31$ to 0.35$)$ \\
\hline 5-week Posttreatment & $85.65 \pm 0.86$ & $0.91 \pm 0.59$ & $86.19 \pm 0.85$ & $0.90 \pm 0.62$ & 0.97 & $0.00(-0.33$ to 0.33$)$ \\
\hline \multicolumn{7}{|l|}{ Sleep Onset Latency, min } \\
\hline Baseline & $19.96 \pm 1.65$ & & $23.55 \pm 1.84$ & & & \\
\hline I-week Posttreatment & $19.73 \pm 1.69$ & $-0.23 \pm 1.25$ & $20.52 \pm 1.86$ & $-3.03 \pm 1.71$ & 0.19 & $-0.22(-0.55$ to 0.11$)$ \\
\hline 5-week Posttreatment & $19.74 \pm 1.70$ & $-0.21 \pm 1.32$ & $21.85 \pm 1.88$ & $-1.70 \pm 1.83$ & 0.52 & $-0.11(-0.44$ to 0.22$)$ \\
\hline \multicolumn{7}{|c|}{ Wake After Sleep Onset, min } \\
\hline Baseline & $35.17 \pm 3.17$ & & $34.09 \pm 3.01$ & & & \\
\hline I-week Posttreatment & $34.62 \pm 3.24$ & $-0.56 \pm 2.51$ & $32.78 \pm 3.03$ & $-1.31 \pm 2.16$ & 0.82 & $-0.04(-0.37$ to 0.29$)$ \\
\hline 5-week Posttreatment & $31.59 \pm 3.22$ & $-3.58 \pm 2.06$ & $30.12 \pm 3.05$ & $-3.97 \pm 2.39$ & 0.91 & $-0.02(-0.35$ to 0.31$)$ \\
\hline \multicolumn{7}{|l|}{ Total Sleep Time, min } \\
\hline Baseline & $394.91 \pm 6.43$ & & $4 \mid 3.35 \pm 6.22$ & & & \\
\hline I-week Posttreatment & $403.10 \pm 6.63$ & $8.19 \pm 6.18$ & $402.74 \pm 6.27$ & $-10.61 \pm 5.14$ & 0.02 & $0.40(0.06$ to 0.73$)$ \\
\hline 5-week Posttreatment & $401.31 \pm 6.63$ & $6.40 \pm 5.63$ & $407.09 \pm 6.34$ & $-6.26 \pm 6.11$ & 0.12 & $0.26(-0.08$ to 0.59$)$ \\
\hline
\end{tabular}

Notes: ${ }^{a}$ Estimated mean and standard error from linear mixed-effects model. ${ }^{\mathrm{b}} \mathrm{P}$-value for group by time interaction of mean score using linear mixed-effects models. ${ }^{\mathrm{c} B e t w e e n-g r o u p ~ e f f e c t ~ s i z e ~ c a l c u l a t i o n ~ w a s ~ b a s e d ~ o n ~ t h e ~ b e t w e e n-g r o u p ~ d i f f e r e n c e ~ i n ~ t h e ~ m e a n ~ c h a n g e s ~ f r o m ~ b a s e l i n e ~ d i v i d e d ~ b y ~ a ~ p o o l e d ~ s t a n d a r d ~ d e v i a t i o n . ~ A ~ p o s i t i v e ~ s i g n ~}$ was used to denote a superior effect in the TA group to the SA group.

Abbreviations: Cl, Confidence Interval; ES, Effect Size; SA, Sham-acupuncture; SE, Standard Error; TA, Traditional-acupuncture.

Table 4 Hospital Anxiety and Depression Scale (HADS) and Short Form Six Dimensions (SF6D) Across Study Time Points

\begin{tabular}{|c|c|c|c|c|c|c|}
\hline \multirow[t]{2}{*}{ Outcomes } & \multicolumn{2}{|c|}{ TA $(N=70)$} & \multicolumn{2}{|c|}{ SA $(N=70)$} & \multirow[t]{2}{*}{ P-value ${ }^{b}$} & \multirow{2}{*}{$\begin{array}{c}\text { Between-Group ES } \\
(95 \% \mathrm{Cl})^{\mathrm{c}}\end{array}$} \\
\hline & $\begin{array}{c}\text { Estimated } \\
\text { Mean } \pm \mathrm{SE}^{\mathrm{a}}\end{array}$ & $\begin{array}{c}\text { Change from Baseline, } \\
\text { Mean } \pm \text { SE }\end{array}$ & $\begin{array}{c}\text { Estimated } \\
\text { Mean } \pm \mathrm{SE}^{\mathrm{a}}\end{array}$ & $\begin{array}{c}\text { Change from Baseline, } \\
\text { Mean } \pm \text { SE }\end{array}$ & & \\
\hline \multicolumn{3}{|c|}{ Hospital Anxiety and Depression Scale (HADS) } & & & & \\
\hline \multicolumn{7}{|l|}{ Anxiety } \\
\hline Baseline & $8.07 \pm 0.43$ & & $8.24 \pm 0.41$ & & & \\
\hline I-week Posttreatment & $6.57 \pm 0.44$ & $-1.50 \pm 0.29$ & $7.20 \pm 0.41$ & $-1.04 \pm 0.32$ & 0.29 & $0.18(-0.15,0.51)$ \\
\hline 5-week Posttreatment & $6.19 \pm 0.44$ & $-1.88 \pm 0.39$ & $6.45 \pm 0.42$ & $-1.80 \pm 0.40$ & 0.88 & $0.02(-0.31,0.36)$ \\
\hline \multicolumn{7}{|l|}{ Depression } \\
\hline Baseline & $6.37 \pm 0.43$ & & $6.51 \pm 0.41$ & & & \\
\hline I-week Posttreatment & $5.57 \pm 0.44$ & $-0.81 \pm 0.29$ & $5.03 \pm 0.41$ & $-1.48 \pm 0.31$ & 0.11 & $-0.27(-0.60$ to 0.07$)$ \\
\hline 5-week Posttreatment & $4.82 \pm 0.44$ & $-1.55 \pm 0.34$ & $5.03 \pm 0.42$ & $-1.49 \pm 0.41$ & 0.90 & $0.02(-0.31$ to 0.35$)$ \\
\hline \multicolumn{7}{|c|}{ Short Form Six Dimensions (SF6D) } \\
\hline Baseline & $0.70 \pm 0.02$ & & $0.69 \pm 0.02$ & & & \\
\hline I-week Posttreatment & $0.74 \pm 0.02$ & $0.04 \pm 0.01$ & $0.74 \pm 0.02$ & $0.05 \pm 0.01$ & 0.69 & $-0.12(-0.45$ to 0.21$)$ \\
\hline 5-week Posttreatment & $0.76 \pm 0.02$ & $0.07 \pm 0.01$ & $0.76 \pm 0.02$ & $0.06 \pm 0.01$ & 0.94 & $0.12(-0.21$ to 0.45$)$ \\
\hline
\end{tabular}

${ }^{a}$ Notes: ${ }^{a}$ Estimated mean and standard error from linear mixed-effects model. ${ }^{b}$ P-value for group by time interaction of mean score using linear mixed-effects models. 'Between-group effect size calculation was based on the between-group difference in the mean changes from baseline divided by a pooled standard deviation. A positive sign was used to denote a superior effect in the TA group to the SA group.es.

Abbreviations: $\mathrm{Cl}$, Confidence Interval; ES, Effect Size; SA, Sham-acupuncture; SE, Standard Error; TA, Traditional-acupuncture. 
Table 5 Oxidative Stress Parameters by Fasting Blood Sample Across Study Time Points

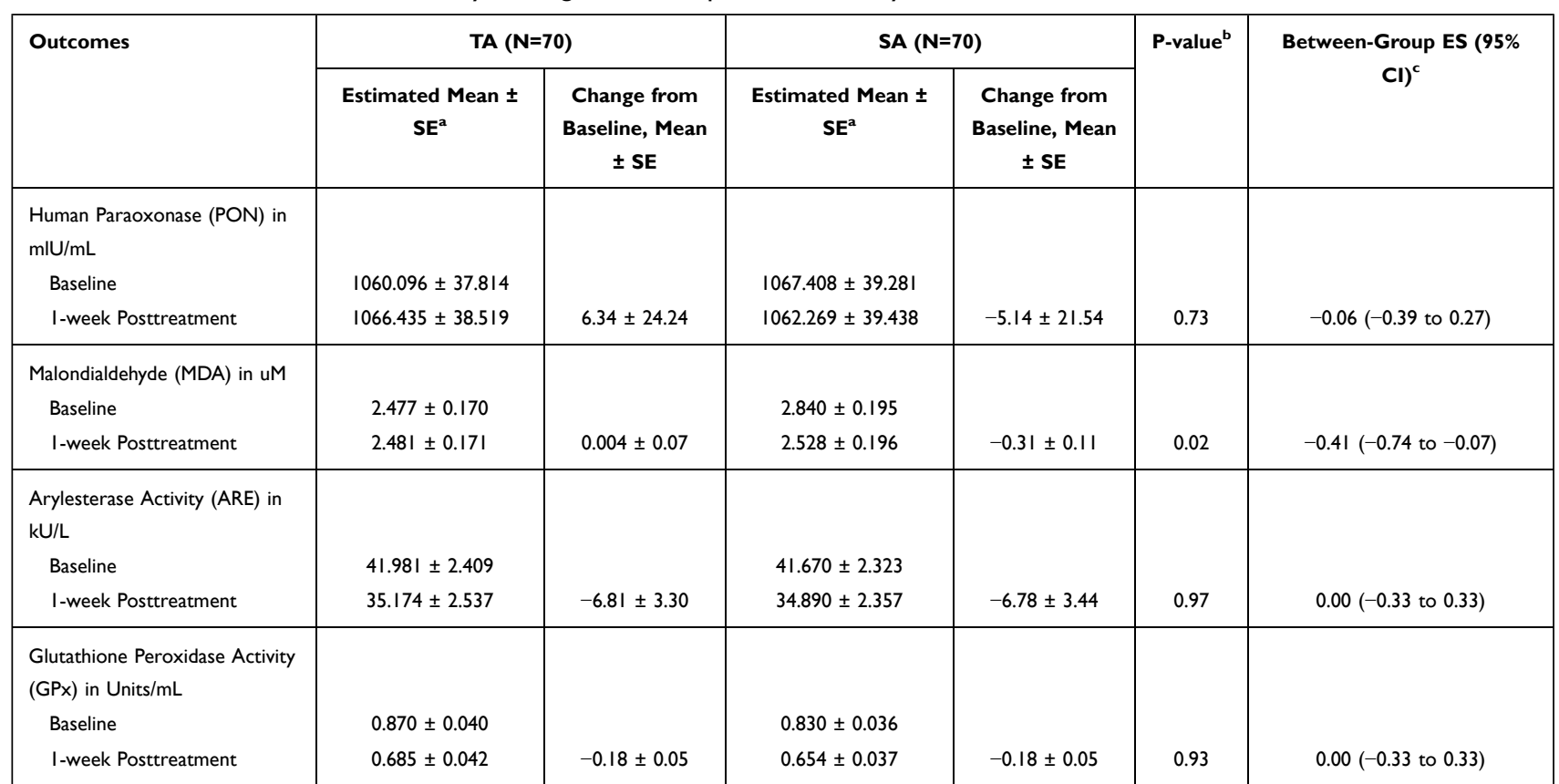

Notes: ${ }^{a}$ Estimated mean and standard error from linear mixed-effects model. ${ }^{b}$ P-value for group by time interaction of mean score using linear mixed-effects models. ${ }^{c}$ Between-group effect size calculation was based on the between-group difference in the mean changes from baseline divided by a pooled standard deviation. A positive sign was used to denote a superior effect in the TA group to the SA group.

Abbreviations: $\mathrm{Cl}$, Confidence Interval; ES, Effect Size; SA, Sham-acupuncture; SE, Standard Error; TA, Traditional-acupuncture.

\section{Success of Blinding and Credibility Assessment}

No significant between-group difference was found in participants' confidence in "recommending the intervention to others" or "relieving other symptoms", but the TA group showed a higher confidence in relieving insomnia symptoms $(p=0.04)$ and the perceived logic of the treatment $(p=0.03)$. The participants were asked to make a guess of their group allocations. There was no significant group difference $(p=0.22)$ in the proportion of participants who guessed that they had received TA (TA vs SA, $45.6 \%$ vs $32.9 \%$ ) or had no idea (TA vs SA, $42.6 \%$ vs $47.1 \%$ ).

\section{Discussion}

Our results found there was no significant difference between TA and SA in improving subjective sleep efficiency, the predefined primary outcome, and most of the other outcomes in individuals with insomnia disorder. However, the TA group showed a longer TST as measured by both sleep diary and actigraphy at 1-week post-treatment; and a higher proportion of participants with a clinical significance of SE $\geq$ $85 \%$ at the 5-week posttreatment follow-up. The findings suggest that non-invasive SA without skin penetration or electric stimulation leads to both subjective sleep and objective sleep improvement, which is comparable to TA in most outcomes. Overall, both TA and SA are generally safe and well-tolerated interventions.

To our knowledge, this is the first sham-controlled RCT to examine a semi-individualized acupuncture based on TCM theory for insomnia disorder and the first RCT to explore the effects of acupuncture on oxidative stress in insomnia. It is also the largest sham-controlled RCT of acupuncture for insomnia. ${ }^{50}$ Our results show that participants in both groups showed sleep improvement in ISI score and sleep-diary-measured SE, although no significant differences were found. The results were contrary to the previous RCTs of acupuncture for primary insomnia using non-invasive sham needles as a control. ${ }^{51,52}$ Yin et al found that the acupuncture group showed a significant increase in sleep-diary-derived SE and an improvement in ISI total score. ${ }^{51}$ Our previous small-scale RCT of acupuncture for insomnia found that the acupuncture group had a significantly higher sleep-diary-derived SE than the SA, but no significant difference in ISI score. The discrepancy may be because more needles were used in our study (16 needles) than in these two studies (8-9 needles), and during each treatment session, our 
acupuncturist provided a 5-10 minute individual TCM consultation to the participants to assess their current sleep, pulse, and symptoms using TCM diagnostic methods. These might have tremendously increased the placebo effect of our intervention, as previous studies found that a higher number of needles ${ }^{52}$ and a warmer interaction ${ }^{53}$ between practitioner and patients would enhance the placebo effects. This resulted in large, non-specific effects, but left little space for the assumed specific effects of acupuncture, resulting in no significant difference between TA and SA in the present study.

It is also worth noting that we only examined a treatment frequency of 2 session per week for a 4-week acupuncture treatment period. Whether a more frequent or longer treatment period would produce better results is remain unknown. A recent systematic review of $15 \mathrm{RCT}$ of acupuncture versus sham acupuncture for insomnia showed that the frequency of acupuncture treatment session ranged from 2 to 7 per week, and the duration of treatment ranged from 3 to 8 weeks. ${ }^{19}$ Further dose-response studies may be conducted to determine the best frequency and duration of the acupuncture treatment. As an indicator of the sufficiency of sleep length, participants who received TA reported a longer total sleep time derived from both subjective and objective measures than those who received SA, although no significant between-group difference was found in sleep efficiency or perceived symptoms severity as measured by ISI. The misalignment between quantity and quality in those with insomnia has been reported by Shrivastava et al, who noted that the presence of fragmented sleep could result in poor sleep quality, even with an adequate sleep duration. ${ }^{54}$ Moreover, sleep quality refers to an overall evaluation of how well an individual slept, which was judged with reference to a wide range of factors. Previous studies indicated that having sufficient sleep was not listed in the first five determinants of perceptions of good sleep in insomnia sufferers, apart from the restorative feeling after sleep, daytime functioning, and the frequency of awakenings at night. ${ }^{55}$

Previous studies reported that individuals with insomnia had higher levels of oxidative stress than good sleepers. ${ }^{26,27}$ Our study explored whether the acupuncture treatment altered the oxidative stress level in individuals with insomnia disorder, since acupuncture has been reported to reduce oxidative stress levels in vascular dementia, Alzheimer's disease, Parkinson's disease, and hypertension. ${ }^{29,56}$ Although we found the TA group showed a longer subjective and objective TST at 1-week post-treatment, there were no significant between-group difference in the oxidative stress parameters, except that, a significant change in MDA level was found between the TA and SA groups. However, the difference was likely due to the higher baseline level in the SA group. Our results showed that the activity of GPx, an anti-oxidative enzyme that protects the organism from oxidative damage, decreased in both TA and SA groups after receiving the intervention. The results suggested that the level of lipid hydroperoxides, a byproduct of oxidative stress, reduced after the acupuncture intervention. Further investigation is needed to confirm the role of oxidative stress in the mechanism of acupuncture for relieving insomnia.

The strengths of this study included the use of noninvasive sham needles to control for placebo effects. All the participants were screened using a home-based polysomnography to exclude those with sleep apnea. We adopted a treatment procedure that was more reflective of the usual clinical practice of acupuncturists, including a TCM four-diagnostic assessment at each treatment visit and semi-individualized acupuncture treatment protocols.

This study also has a number of limitations. First, our study used a semi-individualized prescription with eight fixed standard points plus eight acupuncturist-selected points. Although we allowed the acupuncturists to select the points from a list of commonly used acupoints, the acupuncturists were not allowed to change the standard points or to use acupoints not on the list. Such an approach may not fully resemble the acupuncturists' clinical practice. On the other hand, the semi-individualized approach may not completed replicable in future studies but it ensured that the acupuncture treatment protocol would not vary greatly among participants and enabled replication in some extents in future studies. Second, the participants who voluntarily participated in this study may have had higher expectations of an affinity toward acupuncture, which may have increased their response to the intervention. Third, the present sample consisted of $80 \%$ of female which may limit the generalizability of the finding in male. Finally, we did not include a care as usual group or standard treatment group. Future studies using a pragmatic approach are warranted to compare the effects of different acupuncture approaches on improving sleep.

\section{Conclusions}

TA and SA did not significantly differ in improving subjective sleep efficiency in individuals with insomnia disorder. However, TA showed a short-term effect on improving TST as measured by both sleep diary and actigraphy, but 
there were no differences in the oxidative stress parameters. In terms of safety, both TA and SA were well-tolerated. The findings suggest that non-invasive SA without skin penetration or electric stimulation leads to sleep improvement, which is comparable to TA in most sleep-related outcomes and oxidative stress parameters.

\section{Data Sharing Statement}

The data that support the findings of this study are available from the corresponding author (Wing-fai Yeung) upon reasonable request. Individual- deidentified participant data are available after contacting the corresponding author via email. The data will be available immediately after publication without an end date.

\section{Clinical Trial Registration}

Registry: ClinicalTrials.gov; Title: Acupuncture in the Modulation of Peripheral Oxidative Stress Insomnia; Identifier NCT03447587; URL: https://clinicaltrials.gov/ ct2/show/NCT03447587

\section{Acknowledgments}

This was not an industry-supported study. This project was supported by Research Grants Council of Hong Kong China, Early Career Scheme (Project no.: 25101017). Special thanks went to all the participants. We also thank Ms. Chi Ling Lau and Ms. Ying Lee Lam for delivering the intervention. We were also thankful to those participants who participated in this study.

\section{Disclosure}

The Authors declare that there were no conflicts of interest and no financial assistance was received in support of the study.

\section{References}

1. American Psychiatric Association. Diagnostic and Statistical Manual of Mental Disorders: DSM-5. Arlington, VA: American Psychiatric Association; 2013.

2. Ohayon MM, Reynolds C. Epidemiological and clinical relevance of insomnia diagnosis algorithms according to the DSM-IV and the International Classification of Sleep Disorders (ICSD). Sleep Med. 2009;10(9):952-960.

3. Chung KF, Yeung WF, Ho FY, Yung KP, Yu YM, Kwok CW. Crosscultural and comparative epidemiology of insomnia: the Diagnostic and Statistical Manual (DSM), International Classification of Diseases (ICD) and International Classification of Sleep Disorders (ICSD). Sleep Med. 2015;16(4):477-482.

4. Hertenstein E, Feige B, Gmeiner T, et al. Insomnia as a predictor of mental disorders: a systematic review and meta-analysis. Sleep Med Rev. 2019;43:96-105.
5. Wardle-Pinkston S, Slavish DC, Taylor DJ. Insomnia and cognitive performance: a systematic review and meta-analysis. Sleep Med Rev. 2019;48:101205.

6. Ge L, Guyatt G, Tian J, et al. Insomnia and risk of mortality from all-cause, cardiovascular disease, and cancer: systematic review and meta-analysis of prospective cohort studies. Sleep Med Rev. 2019;48:101215.

7. Perlis ML, Grandner MA, Chakravorty S, Bernert RA, Brown GK, Thase ME. Suicide and sleep: is it a bad thing to be awake when reason sleeps? Sleep Med Rev. 2016;29:101-107.

8. Taylor DJ, Lichstein KL, Durrence HH. Insomnia as a health risk factor. Behav Sleep Med. 2003;1(4):227-247.

9. Yeung WF, Chung KF, Wong CY. Relationship between insomnia and headache in community-based middle-aged Hong Kong Chinese women. J Headache Pain. 2010;11(3):187-195.

10. Neckelmann D, Mykletun A, Dahl AA. Chronic insomnia as a risk factor for developing anxiety and depression. Sleep. 2007;30 (7):873-880. doi:10.1093/sleep/30.7.873

11. Sateia MJ, Buysse DJ, Krystal AD, Neubauer DN, Heald JL. Clinical practice guideline for the pharmacologic treatment of chronic insomnia in adults: an American academy of sleep medicine clinical practice guideline. J Clin Sleep Med. 2017;13(2):307-349. doi:10.5664/jcsm.6470

12. Sweetman A, Lovato N, Micic G, et al. Do symptoms of depression, anxiety or stress impair the effectiveness of cognitive behavioural therapy for insomnia? A chart-review of 455 patients with chronic insomnia. Sleep Med. 2020;75:401-410.

13. van der Zweerde T, Bisdounis L, Kyle SD, Lancee J, van Straten A. Cognitive behavioral therapy for insomnia: a meta-analysis of long-term effects in controlled studies. Sleep Med Rev. 2019;48:101208.

14. Edinger JD, Sampson WS. A primary care "friendly" cognitive behavioral insomnia therapy. Sleep. 2003;26(2):177-182.

15. Bertisch SM, Wells RE, Smith MT, McCarthy EP. Use of relaxation techniques and complementary and alternative medicine by American adults with insomnia symptoms: results from a national survey. J Clin Sleep Med. 2012;8(6):681-691.

16. Chung KF, Yeung WF, Ho FY, et al. Validity and reliability of the Brief Insomnia Questionnaire in the general population in Hong Kong. J Psychosom Res. 2014;76(5):374-379.

17. Kim SH, Jeong JH, Lim JH, Kim BK. Acupuncture using pattern-identification for the treatment of insomnia disorder: a systematic review and meta-analysis of randomized controlled trials. Integrative Med Res. 2019;8(3):216-226.

18. Yeung WF, Chung KF, Leung YK, Zhang SP, Law AC. Traditional needle acupuncture treatment for insomnia: a systematic review of randomized controlled trials. Sleep Med. 2009;10(7):694-704.

19. Zhang J, He Y, Huang X, Liu Y, Yu H. The effects of acupuncture versus sham/placebo acupuncture for insomnia: a systematic review and meta-analysis of randomized controlled trials. Complement Ther Clin Pract. 2020;41:101253.

20. Cheuk DK, Yeung WF, Chung KF, Wong V. Acupuncture for insomnia. Cochrane Database Syst Rev. 2007;3:Cd005472.

21. Cheuk DK, Yeung WF, Chung KF, Wong V. Acupuncture for insomnia. Cochrane Database Syst Rev. 2012;9:CD005472.

22. Jiang M, Lu C, Zhang C, et al. Syndrome differentiation in modern research of traditional Chinese medicine. $J$ Ethnopharmacol. 2012;140(3):634-642.

23. Sies H. Role of reactive oxygen species in biological processes. Klin Wochenschr. 1991;69(21-23):965-968.

24. Palagini L, Geoffroy PA, Miniati M, et al. Insomnia, sleep loss, and circadian sleep disturbances in mood disorders: a pathway toward neurodegeneration and neuroprogression? A theoretical review. CNS Spectr. 2021;1-11. doi:10.1017/S1092852921000018

25. Villafuerte G, Miguel-Puga A, Rodriguez EM, Machado S, Manjarrez E, Arias-Carrion O. Sleep deprivation and oxidative stress in animal models: a systematic review. Oxid Med Cell Longev. 2015;2015:234952. doi:10.1155/2015/234952 
26. Gulec M, Ozkol H, Selvi Y, et al. Oxidative stress in patients with primary insomnia. Prog Neuropsychopharmacol Biol Psychiatry. 2012;37(2):247-251. doi:10.1016/j.pnpbp.2012.02.011

27. Liang B, Li YH, Kong H. Serum paraoxonase, arylesterase activities and oxidative status in patients with insomnia. Eur Rev Med Pharmacol Sci. 2013;17(18):2517-2522.

28. Hachul de Campos H, Brandao LC, D'Almeida V, et al. Sleep disturbances, oxidative stress and cardiovascular risk parameters in postmenopausal women complaining of insomnia. Climacteric. 2006;9(4):312-319.

29. Zeng XH, Li QQ, Xu Q, Li F, Liu CZ. Acupuncture mechanism and redox equilibrium. Evid Based Complement Alternat Med. 2014;2014:483294. doi:10.1155/2014/483294

30. Bastien $\mathrm{CH}$, Vallières A, Morin CM. Validation of the Insomnia Severity Index as an outcome measure for insomnia research. Sleep Med. 2001;2(4):297-307.

31. Morin CM, Belleville G, Bélanger L, Ivers H. The Insomnia Severity Index: psychometric indicators to detect insomnia cases and evaluate treatment response. Sleep. 2011;34(5):601-608

32. Erman MK, Stewart D, Einhorn D, Gordon N, Casal E. Validation of the ApneaLink for the screening of sleep apnea: a novel and simple single-channel recording device. J Clin Sleep Med. 2007;3:387-392.

33. Chung KF, Yeung WF, Yu YM, et al. Acupuncture for residual insomnia associated with major depressive disorder: a placebo- and sham-controlled, subject- and assessor-blind, randomized trial. J Clin Psychiatry. 2015;76(6):e752-60.

34. Yeung WF, Chung KF, Zhang SP, Yap TG, Law AC. Electroacupuncture for primary insomnia: a randomized controlled trial. Sleep. 2009;32(8):1039-1047.

35. Yeung WF, Chung KF, Zhang ZJ, et al. Electroacupuncture for tapering off long-term benzodiazepine use: a randomized controlled trial. J Psychiatr Res. 2019;109:59-67.

36. Yeung WF, Chung KF, Poon MM, et al. Prescription of chinese herbal medicine and selection of acupoints in pattern-based traditional chinese medicine treatment for insomnia: a systematic review. Evid Based Complement Alternat Med. 2012;2012:902578.

37. Poon MMK, Chung KF, Yeung WF, Yau VHK, Zhang SP. Classification of insomnia using the traditional Chinese medicine system: a systematic review. Evid Based Compl Altern Med. 2012;2012:735078. doi:10.1155/2012/735078

38. Streitberger K, Kleinhenz J. Introducing a placebo needle into acupuncture research. Lancet. 1998;352(9125):364-365.

39. Carney CE, Buysse DJ, Ancoli-Israel S, et al. The consensus sleep diary: standardizing prospective sleep self-monitoring. Sleep. 2012;35(2):287-302.

40. Zigmond AS, Snaith RP. The hospital anxiety and depression scale. Acta Psychiatr Scand. 1983;67(6):361-370.

41. Brazier J, Roberts J, Deverill M. The estimation of a preference-based measure of health from the SF-36. J Health Econ. 2002;21(2):271-292.
42. Wong CKH, Mulhern B, Cheng GHL, Lam CLK. SF-6D population norms for the Hong Kong Chinese general population. Qual Life Res. 2018;27(9):2349-2359.

43. Chung KF, Kan KK, Yeung WF. Assessing insomnia in adolescents: comparison of Insomnia Severity Index, Athens Insomnia Scale and Sleep Quality Index. Sleep Med. 2011;12(5):463-470.

44. Leung CM, Wing YK, Kwong PK, Lo A, Shum K. Validation of the Chinese-Cantonese version of the hospital anxiety and depression scale and comparison with the Hamilton Rating Scale of Depression. Acta Psychiatr Scand. 1999;100(6):456-461.

45. Lam CLK, Brazier J, McGhee SM. Valuation of the SF-6D health states is feasible, acceptable, reliable, and valid in a Chinese population. Value Health. 2008;11(2):295-303.

46. Marino M, Li Y, Rueschman MN, et al. Measuring sleep: accuracy, sensitivity, and specificity of wrist actigraphy compared to polysomnography. Sleep. 2013;36(11):1747-1755.

47. Chung KF, Yeung WF, Yu YM, Kwok CW, Zhang SP, Zhang ZJ. Adverse events related to acupuncture: development and testing of a rating scale. Clin J Pain. 2015;31(10):922-928.

48. Vincent C. Credibility assessment in trials of acupuncture. Complementary Medical Research. 1990;4:8-11.

49. Cohen J. Statistical Power Analysis for the Behavioral Sciences. Hillsdale, NJ: Lawrence Earlbaum Associates; 1988.

50. Cao HJ, Yu ML, Wang LQ, Fei YT, Xu H, Liu JP. Acupuncture for primary insomnia: an updated systematic review of randomized controlled trials. J Altern Complement Med. 2019;25(5):451-474.

51. Yin X, Gou M, Xu J, et al. Efficacy and safety of acupuncture treatment on primary insomnia: a randomized controlled trial. Sleep Med. 2017;37:193-200.

52. MacPherson H, Maschino AC, Lewith G, et al. Characteristics of acupuncture treatment associated with outcome: an individual patient meta-analysis of 17,922 patients with chronic pain in randomised controlled trials. PLoS One. 2013;8(10):e77438.

53. Kaptchuk TJ, Kelley JM, Conboy LA, et al. Components of placebo effect: randomised controlled trial in patients with irritable bowel syndrome. BMJ. 2008;336(7651):999-1003.

54. Shrivastava D, Jung S, Saadat M, Sirohi R, Crewson K. How to interpret the results of a sleep study. J Community Hosp Intern Med Perspect. 2014;4:24983.

55. Harvey AG, Stinson K, Whitaker KL, Moskovitz D, Virk H. The Subjective Meaning of Sleep Quality: a comparison of individuals with and without Insomnia. Sleep. 2008;31(3):383-393.

56. Li ZR, Shen MH, Peng YJ. Progress in researches on the effect of acupuncture in antagonizing oxygen stress. Chin J Integr Med. 2005;11(2):156-160.
Nature and Science of Sleep

\section{Publish your work in this journal}

Nature and Science of Sleep is an international, peer-reviewed, open access journal covering all aspects of sleep science and sleep medicine, including the neurophysiology and functions of sleep, the genetics of sleep, sleep and society, biological rhythms, dreaming, sleep disorders and therapy, and strategies to optimize healthy sleep.
The manuscript management system is completely online and includes a very quick and fair peer-review system, which is all easy to use. Visit http://www.dovepress.com/testimonials.php to read real quotes from published authors. 